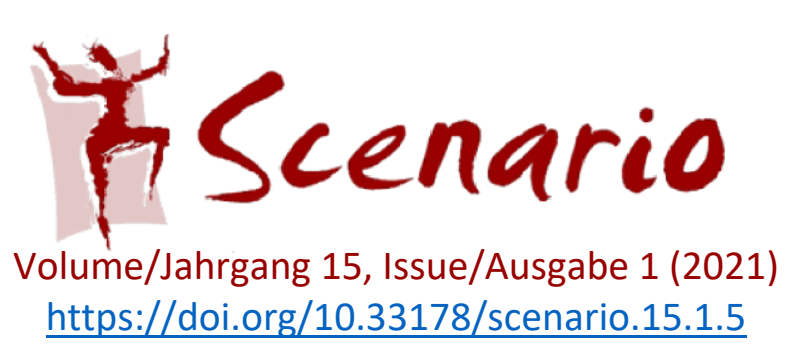

\title{
Meine neue Stimme
}

\section{Chorisches \\ Sprechen \\ zur \\ Stärkung \\ der}

Sprachbewusstheit und Selbstwahrnehmung in der

Deutschlehrer:innenbildung

\section{Lena Merkle}

Die Stimme einer Lehrperson ist ein zentrales pädagogisches Instrument. Fremdsprachenlehrende sind - zumindest im klassischen Unterricht - zudem Sprachvorbilder im kommunikativen und auch ästhetischen Sinne. Jede Stimme hat einen individuellen Klang, der plastizierbar ist und zielgerichtet eingesetzt werden kann, um ein konstruktives Lernklima zu schaffen. In diesem Beitrag wird ein Seminarmodul für auszubildende DaF-Lehrende vorgestellt, das auf performativ-ästhetischen Arbeitsansätzen basiert. Die Arbeitsform des chorischen Sprechens fand bisher vor allem in der muttersprachlichen und fremdsprachlichen Schulbildung zur Stärkung sprachlich-ästhetischer und persönlichkeitsbildender Kompetenzen Anwendung. Mit ähnlicher Zielsetzung soll im vorliegenden Beitrag aufgezeigt werden, dass der persönliche stimmliche Ausdruck, unter Einbezug des Körperlichen, ein ernstzunehmendes Arbeitsfeld in der fremdsprachlichen Lehrer:innenbildung ist.

Ich fürchte mich so vor der Menschen Wort. Sie sprechen alles so deutlich aus:

Und dieses heißt Hund und jenes heißt Haus, und hier ist Beginn und das Ende dort.

Mich bangt auch ihr Sinn, ihr Spiel mit dem Spott, sie wissen alles, was wird und war; kein Berg ist ihnen mehr wunderbar; ihr Garten und Gut grenzt grade an Gott.

Ich will immer warnen und wehren: bleibt fern. Die Dinge singen hör ich so gern. Ihr rührt sie an: Sie sind starr und stumm. Ihr bringt mir alle die Dinge um.

Rainer Maria Rilke (1898)

\section{Problemaufriss}

In der Lehrer:innenbildung wird der Blick zunehmend auf die persönlichkeitsbezogene Kompetenzentwicklung gerichtet (Haack, 2018). Damit verbunden sind u. a. Selbstmotivation, Selbstwahrnehmung, Bereitschaft zu Kooperation und Problemlösungsfähigkeit im zukünftigen Beruf, die Teil der Entwicklung einer professionellen Lehridentität sind. Seit rund 
20 Jahren rückt dieser Bereich in der internationalen Lehrer:innenbildung mehr und mehr in den Vordergrund und es wird zunehmend anerkannt, dass Lehrende weitaus mehr zu leisten haben als Fachinhalte zu vermitteln (Haack, 2018, S. 11-19). Die Entwicklung des Selbstkompetenzbereichs ist nicht zu unterschätzen, denn mit ihr steht und fällt die eigentliche Arbeitsbereitschaft, die Arbeitsfreude und die Wahrscheinlichkeit, dass die Arbeitskraft der Lehrperson beständig bleibt.

Der Frage nach persönlichkeitsbezogenen Kompetenzen widmet sich auch der Gemeinsame Europäische Referenzrahmen (Europarat, 2001, fortan GeR), darin beschrieben als „Einstellungen und Eigenheiten von Menschen ..., die man beim Sprachenlernen und -lehren in Rechnung stellen muss. " (Absatz 5). Heute, bald 20 Jahre später, wird das Verhältnis zu Sprachen und der Aushandlungsprozess mit ihnen wissenschaftlich wieder aufgegriffen. Dem Begleitband des GeR (2020) ist zu entnehmen, dass sich die klassischen vier Grundfertigkeiten als unzureichend erweisen und Sprachaktivität deshalb durch "makro-funktionale“ Dimensionen erweitert werden muss (S. 38-39). Innerhalb dieser Dimensionen finden sich Vorschläge zur kreativen Auseinandersetzung mit Sprache, die darauf hindeuten, dass der kreativ-produktive Sprachgebrauch in Zukunft an Bedeutung gewinnt. Da Lehrende immer selbst Lernende der facheigenen Fremdsprache sind, kann überdies an die längst sorgfältig ausgearbeiteten persönlichkeitsbezogenen Zielkompetenzen des GeR (2001) angeknüpft werden. Auf diesen formalsprachlichen Grundlagen basierend, soll der Hypothese nachgegangen werden, dass sich Lehrende der stimmlichen Gestalt der Fremdsprache (ihres Faches) nähern können, indem der persönliche Zugang zur Fremdsprache in den Blick genommen wird.

Eine Möglichkeit sich der Sprache affektiv zu nähern, ist die nur schwer zu fassende, als prozessbezogene Kompetenz ausgewiesene Sprachbewusstheit, welche ursprünglich für Fremdsprachenlernende formuliert wurde (GeR, 2001; Hekkala, 2019). In diesem Beitrag wird die Gefühlsseite von Sprachbewusstheit fokussiert und auf Lehrende bezogen.

Durch die enge Verwobenheit von Sprache und Identität (Sapir-Whorf-Hypothese) ist davon auszugehen, dass Sprachbewusstheit zugleich auch Selbstbewusstheit einschließt. Diese beiden Kompetenzfelder sind im Grunde genommen untrennbar und könnten durch eine (Selbst-)Wahrnehmungsschulung herausgearbeitet werden. Innerhalb dieses sprachbezogenen Selbstwahrnehmungsprozesses werden klangliche Besonderheiten und Qualitäten der Sprache erkannt und zugleich die Einstellung zur Sprache deutlich.

Es bleibt allerdings die Frage, wie Impulse für solche Sprachbewusstheit fördernde Erfahrungen gegeben werden können, damit angehende Lehrende eine feinsinnige, 
selbstbewusste und nicht zuletzt begeisterungsfähige Haltung gegenüber dem eigenen Fach und somit auch gegenüber ihrem Beruf entwickeln?

Um auf die Antwort dieser Frage hinzuführen, soll nochmals betont werden, dass Fremdsprachenlehrende eine Art Identitätssuche durchlaufen, die durch ästhetische Bildungsprozesse angeregt werden kann. Dies wurde bereits in einigen Studien gezeigt (Haack, 2018; Schewe, 1993). Die Stimme wiederum ist Ausdruck der Identität, oder, um es mit Boltes (2007) Worten zu sagen: „Mit der Stimme meldet er [der Mensch] sich auf dieser Welt an und er verlässt sie mit dem letzten Atemhauch." (S. 1). Die Bedeutung der Stimme im pädagogischen Kontext und die "stimmlichen Leistungen“ (Hans, 2018, S. 182) einer Lehrperson werden in der Bildungsforschung viel $\mathrm{zu}$ wenig beleuchtet. Hans, selbst Sprachgestalterin, weist mit Recht darauf hin, dass „eine tragfähige, belastbare und variabel einsetzbare Stimme notwendig ist, um das Unterrichtsgeschehen wirkungsvoll führen zu können und angenehme und arbeitsfördernde Bedingungen zu schaffen" (S. 182). Schewe (2014) plädiert konkret für den Einsatz der sogenannten Sprachgestaltung im Laufe der Ausbildung von DaF-Lehrkräften, um die „ästhetische Seite von Sprache“ zu erfassen und „einen Sinn für Sprache entfalten zu können“ (S. 173). Zweifellos ist, dass der dreigliedrige Mensch $^{1}$ die Sprache nicht nur kognitiv erfasst, wenn er von einer Lehrperson lernt, die die Sprache in ganzheitlicher Form erfasst hat.

In diesem Beitrag sollen Anregungen gegeben werden, wie durch chorisches Sprechen Sprachbewusstheit in Bezug auf die Sprechstimme und gleichzeitig die Persönlichkeitsbildung von DaF-Lehramtsstudierenden gefördert werden können. Ein solcher Ansatz wäre in der Dramapädagogik zu verorten und bezieht sich vor allem auf die pädagogische Sprachgestaltung (Hans, 2018).

\section{Chorisches Sprechen im Rahmen der Ausbildung von Fremdsprachenlehrer:innen an der Universität Marmara (Istanbul)}

Das chorische Sprechen wurde in einem Seminarmodul für DaF-Studierende an der Marmara Universität (Istanbul) erprobt. Das Modul wurde in erster Linie mit dem Ziel entwickelt, ästhetische Wahrnehmungs- und Erfahrungsräume mit der Sprache zu eröffnen und somit einen persönlichkeitsbezogenen Zugang zur Fremdsprache herzustellen.

In der akademischen Ausbildung der Fremdsprachenlehrenden wird ein hohes Niveau der kommunikativen Kompetenzen vorausgesetzt. Der Sprung vom inhaltorientierten und (halb)-

\footnotetext{
${ }^{1}$ Hier wird auf den Reformpädagogen Rudolf Steiner Bezug genommen, der den Begriff des ganzheitlichen Lernens einschlägig vorgeprägt hat. Steiner (1983) benutzt dabei das Wort dreigliedrig, um das Zusammenwirken der Bereiche Denken, Fühlen und Wollen hervorzuheben.
} 
gesteuerten schulischen Lernern zum reflektierten, kritischen und ästhetischen Umgang mit der Fremdsprache, stellt oft eine große Herausforderung dar. Einen leichteren Übergang zu den vier Grundfertigkeiten ermöglichen curriculare Vertiefungsseminare. In ihrer Zielsetzung schließen sie zwar an die Entfaltung der kommunikativen Teilkompetenzen auf höheren Niveaustufen an (siehe z.B. Anadolu Üniversitesi), bieten jedoch kaum Möglichkeit für einen reflexiven Sprachlernprozess.

Außerdem ist eine ,Verkopfung' zu beobachten, die auf einseitige, wissensbezogene Lernerfahrungen aus der Schulzeit zurückgeführt werden kann und häufig mit massiven Sprechhemmungen verbunden ist, aus denen sich Lernende mühsam befreien müssen. Auch tun sich Studierenden mit dem produktiven und interaktionalen Sprachgebrauch schwer. Kritisches Lesen und Diskutieren, offene imaginative Aufgaben sind ihrerseits kaum zu bewältigen. Auf dieser Stufe kommt einerseits wieder das sprachliche (Selbst)-Bewusstsein ins Spiel, mit welchem Weichen für einen flexiblen und identifikativen Umgang mit der Sprache gestellt werden können, andererseits auch ein frischer Blick auf die klangliche Form und den persönlichen stimmlichen Ausdruck. Dieser Arbeitsschritt - von der Schüler:innenstimme zur Lehrer:innenstimme hin - wird im vorliegenden Beitrag beleuchtet und die praktische Umsetzung vorgestellt. Im Folgenden soll dargelegt werden, auf welchen theoretischen Grundannahmen die Modulausarbeitung fußt, die im Abschnitt 2.2 detaillierter dargestellt wird. Die Bedeutung der Kernbegriffe Sprachbewusstheit, Sprachgestaltung und Dramapädagogik wird geklärt und deren Verbindung zum chorischen Sprechen aufgezeigt.

\subsection{Zur theoretischen Fundierung chorischen Sprechens im Rahmen eines neuen Seminarmoduls}

Um Sprachbewusstheit für eine (neue) Sprache zu entwickeln, sollten eigene Ressourcen mobilisiert werden und Lernende sollten sich auch mit Hilfe von Lernstrategien die Sprache zu Eigen machen (GeR, 2001, S. 62). Dieser Lernschritt wird in der Sprachenlehre als autonomes Lernen bezeichnet. Dies gilt es sich als einen emanzipatorischen Prozess vorzustellen, in dem Lernende in vielfältiger Weise mit der Sprache umgehen lernen und sich mit ihr immer mehr verbinden. Metakognitive Strategien helfen dabei, individuelle Wege zu finden, den Sprachenlernprozess zu steuern und zu kontrollieren. Auffallend ist, dass Sprachbewusstheit angeblich in erster Linie durch linguistisches Wissen sowie sprachliches Handlungswissen erreicht werden kann (Eichler \& Nold, 2007). Doch im Bereich der o. g. eigenen Ressourcen, kann auch ein Sprachgefühl vermutet werden. Wird nun das Sprachgefühl als sprachästhetische Kompetenz gesehen, sollte diese - wie anhand des Praxisbeispiels in Abschnitt 2.2 dargestellt wird - vor allem in der Lehrer:innenbildung, nachdem linguistischformale Strukturen gefestigt worden sind, eine wichtige Rolle spielen. 
Als Teilkompetenz definiert, beeinflusst Sprachbewusstheit fast alle anderen Bereiche im Sprachlernprozess (Eichler \& Nold, 2007). Gerade im Studium der Fremdsprachendidaktik könnte auf dieser metasprachlichen Ebene gearbeitet werden, indem ästhetische Erfahrungen unter Einbezug von Literatur angeregt werden. Auf die Verankerung der Sprachbewusstheit im GeR (2001) wurde schon eingangs hingewiesen. Als brandneu mitberücksichtigte Fertigkeit wurde der Umgang mit literarischen Texten nun auch im Ergänzungsband mit Deskriptoren versehen, wodurch der Umgang mit künstlerisch-ästhetischer Sprache aufgewertet, systematisiert und auch Evaluationskriterien angeboten werden (GeR, 2020, S. 126-129).

Eine solche sprachreflexive und künstlerisch-praktische Arbeit mit Sprache findet sich sozusagen als ,Methodik des Sprechchores' in der pädagogischen Sprachgestaltung (creative speech). Diese vor über 100 Jahren von Rudolf Steiner und Marie Steiner-von Sivers (1981) begründete erzieherische Kunstform, damals noch nicht wissenschaftlich belegbar, hat eine lange Lehrtradition an Waldorfschulen weltweit. Sie wurde von der langjährigen Sprachgestalterin und Lehrerin Christa Slezak-Schindler (2007) federführend weiterentwickelt und ist fester Bestandteil der Lehrer:innenbildung für Waldorfschulen. Anzumerken ist, dass „in der Waldorfpädagogik ... künstlerischer Orientierung ein hoher Stellenwert beigemessen [wird]. Von ihr könnten wichtige Impulse für die Praxis von Deutsch als Fremdsprache ausgehen" (Schewe, 2014, S. 171). Die pädagogische Sprachgestaltung betont die enge Verbindung zwischen Sprache und Persönlichkeit und wirft ein neues Licht auf den Bereich der Sprecherziehung und naturwissenschaftlich ausgerichteten angewandten Phonetik. Diese ist vornehmlich auf metakognitive Reflexion ausgerichtet und führt "nicht automatisch zur Entwicklung lebendigeren, persönlichen Ausdrucks in der Fremdsprache" (Bolte, 2007, S. 4). Im Vergleich zur Ausspracheschulung für Lernende, zu der es neu ausdifferenzierte Skalen gibt (GeR, 2020, S. 159), wird in der Lehrer:innenbildung ein erweiterter Ansatz benötigt. Der Entwicklung eines solchen Ansatzes nachgehend sowie Schewes (1993) Feststellung, dass „verbale und körperliche Ausdrucksfähigkeit“ (S. 426) in der Lehrer:innenbildung vernachlässigt wird, legen es nahe, neue methodische Ansätze im Kontext der angewandten Sprechwissenschaft zu erproben. Von sprachgestalterischen Ansätzen könnten dabei wertvolle Impulse ausgehen.

Sprachgestaltung basiert auf dramapädagogischen Arbeitsweisen, im Fokus ist - ganz im Sinne von Steiners Überlegungen - der „ganze Mensch“ (Haack, 2018, S. 49):

Es ist eben nötig ..., daß der Schauspieler seinen eigenen Körper gut kennenlernt, denn diese eigene Körperlichkeit ist im Grunde genommen für den wirklichen Menschen, der zu spielen hat, das Instrument, auf dem er spielt. Er muß seinen eigenen Körper so kennenlernen wie der Violinspieler seine Geige; die muß er kennen. Er muß gewissermaßen in der Lage sein, 
seiner eigenen Stimme zuzuhören. Man kann das. Man kann es allmählich dahin bringen, daß man seine eigene Stimme immer so, wie wenn sie einen umwellte, hört. Das muß man aber üben, indem man etwa dramatische, es können auch lyrische sein, aber sehr stark in Form, Rhythmus und Takt lebende Verse versucht zu sprechen, ... . (Steiner \& Steiner-von Sivers, 1981, S. 20)

Steiner weist, in bildhafter Sprache, auf die zentrale Beziehung zwischen Sprechen/Stimme und Körperlichkeit hin (vgl. auch aus aktuelleren Werken der Dramapädagogik bei Lutzker, 2007, S. 170; Haack, 2018; zum allgemeinen Sprachenlernen bei Sambanis \& Walter, 2019). Außerdem macht er auf den bildenden Wert ästhetischer Texte und auf die Fähigkeit des, SichSelbst-(Zu)Hörens' aufmerksam. Diese Fähigkeit lässt sich auch in der Theaterarbeit wiederfinden. Nach Haack (2018) ist sie insbesondere für Junglehrer:innen hilfreich, da sie zu mehr Sicherheit im „Einsatz der Fremdsprache als Interaktions- und Lehrersprache“ (S. 65) führe. Die sprachgestaltende Arbeit an Waldorf-Universitäten konzentriert sich auf die Ausbildung einer gesunden Sprechstimme, da die Stimme der Lehrenden als Sprechvorbilder sich unbewusst auf die Sprache der Schüler auswirkt (Hans, 2018). Dies wird durch Atemtechniken, Lautformungs- und Artikulationsübungen sprechkünstlerisch in der Praxis umgesetzt. Hans'These, dass die Qualität des stimmlichen Ausdrucks der Lehrperson den Zugang zur Sprache für den Schüler beeinflusst, lässt sich vom muttersprachlichen Unterricht auf den fremdsprachlichen Unterricht übertragen. Denn, unabhängig vom Alter, sind Fremdsprachenlernende meist im Unterricht in einer Imitationssituation, die von wertvollem rezeptivem Input abhängig ist.

Im sprachgestaltenden Arbeitsprozess verlässt der Sprecher das alltagsfunktionale Verstandeswort, mit dem Ziel "stärker in die Lebendigkeit des Rhythmus und des Atemstroms“ (Slezak-Schindler, 2007, S. 5.) zu kommen. In der Lehrer:innenbildung - ob erstsprachlich oder fremdsprachlich - kann dadurch ein profundes Erfassen der Sprache gelingen und die Sprache selbst als bildhafter Gestaltungsraum erfahren werden. Das ästhetische Erfassen und Gestalten der Sprache fördert zum einen den sprachlichen Ausdruck, zum anderen „löst sich in uns eine Begeisterungsfähigkeit für die Schönheiten der Sprache, die auf die Schüler übergeht" (S. 5). Dadurch kann die sprachgestalterisch ausgebildete Lehrkraft zu einem konstruktiven, motivierenden Lernklima beitragen, in dem Schüler:innen das Atmen und Sprechen mit Wohlempfinden verbinden.

Das chorische Sprechen gehört zur langjährigen Praxis der Sprachgestaltung, insbesondere unter sprachbildnerischem und pädagogischem Gesichtspunkt in der erstsprachlichen Schulbildung (Slezak-Schindler, 2007). Das chorische Theater (Kirchner, 2016) ist in den Theaterwissenschaften zu verorten und hat das Potential, „das Verhältnis zwischen 
Individuum und Gemeinschaft ... sowohl auf der fiktiven als auch auf der spielpraktischen Ebene" (S. 2) zu stärken, sprich es fördert sozial-emotionale Kompetenzen. Auch werden Sprechchöre, unter dem Paradigma des aktiven, handlungsorientierten Ansatzes, im fremdsprachlichen Unterricht nicht selten eingesetzt, um Sprechhemmungen abzubauen (vgl. z.B. Sambanis \& Pickert, 2009) und für Aussprache, Klang und Rhythmus zu sensibilisieren (vgl. z.B. Böttger, 2005). Die mechanischen Modelldialoge kritisierend, nutzt Bolte (2007) das "orchestrierte Sprechen“ im DaF/DaZ-Unterricht, um mit den Lernenden an ihrem persönlichen stimmlichen Ausdruck zu arbeiten. Boltes Beitrag kommt damit der Zielsetzung des vorliegenden Beitrages am nächsten, nur dass hier das chorische Sprechen in der tertiären Bildung ausprobiert und untersucht wird.

Lehrende haben es immer mit Lerngemeinschaften, mit einem kollaborativen Ganzen zu tun. Die Chorkonstellation verfügt über ein vergleichbares Bedingungsgefüge (siehe speziell dazu die Ausführungen zum Aspekt Ensemble im Abschnitt 2.2.2). In einem Chor zu sprechen bedeutet, "die Wirksamkeit der Sprache im zwischenmenschlichen Kontakt" (Waldorfseminar Berlin, o. J., Absatz 2) auszuprobieren. Einzelsprechübungen hingegen sind als vorbereitender Schritt miteingebunden.

Die Sprechchorarbeit, als performativ-ästhetische Aktivität, kann der Dramapädagogik zugeordnet werden. Ihr liegt ein ganzheitliches Menschenbild zu Grunde (Schewe, 1993) und der unvergleichliche Erfahrungsraum, der durch „experimentierendes sprachliches und körperliches Handeln in fiktiven dramatischen Situationen“ (Moraitis, 2011, S. 12) entsteht, wurde bereits in vielen Schulfächern entdeckt. Performativ-ästhetische Aktivität ist ein handlungsbasiertes, kreatives und Selbsterfahrung evozierendes Unternehmen, das vor über zwanzig Jahren in die Fremdsprachendidaktik Einzug gefunden hat. Eine kontroverse wissenschaftliche Diskussion über die Wirksamkeit besteht zwar weiterhin, doch wurde durch zahlreiche empirische Untersuchungen eine deutliche Kompetenzentwicklung bei Lernenden untermauert (ein Überblick dazu in Fleiner, 2016, S. 20-22).

Zum Einsatz performativ-ästhetischer Arbeitsweisen in der Lehrer:innenbildung finden sich bislang allerdings nur vereinzelte Studien, z.B. von Lutzker (2007). In seiner Langzeitstudie, in der er untersucht, welche Wirkung Clowning- und Improvisationsworkshops im Rahmen der Ausbildung von Englischlehrer:innen hatten, kommt er zu folgendem Ergebnis:

The clowning and improvisation courses ... have had significant effects on their personal and professional development. Such development may have included and enhanced openness and attentiveness, a heightened sense of empathy, a higher degree of presence and increased improvisational skills. At the same time, these courses have also played an important role in helping teachers to learn to address their own uncertainties, anxieties and 
mistakes in a more constructive and creative manner. It is postulated that this development may have occurred both in short-term as well as in longterm contexts. (S. 102)

Innerhalb des Selbstkompetenzrasters (Unterweger, 2014, S. 5-10) für Pädagog:innen, kristallisieren sich in Lutzkers Studie Erfolge in unterschiedlichen Selbstkompetenz-Bereichen heraus: Im Motivations- und Emotionsbereich konnte an Unsicherheiten, Fehlern und sogar Ängsten gearbeitet werden. Im Bereich der Selbstwahrnehmung, Selbstreflexion und Kommunikation konnten Selbstpräsenz gestärkt sowie Selbstausdruck und Handlungsstrategien für unvorhersehbare Situationen (Improvisation) entwickelt werden.

In der Hochschulbildung für Fremdsprachenlehrende gibt es noch keine wissenschaftlich dokumentierten und empirisch unterlegten Studien zur Wirksamkeit sprechchorischer bzw. sprachgestaltender Arbeitsformen. Dieser Beitrag soll als Anregung und Konzeptvorschlag dienen, mit Studierenden an ihrem sprachlichen und stimmlichen Ausdruck zu arbeiten, damit sie sich darin verbessern und Sprachphänomene bewusster wahrnehmen.

\subsection{Phasen des innovativen Seminarmoduls Chorisches Sprechen}

Das Seminarmodul war integriert in die curriculare Lehrveranstaltung mit dem Titel Sprechkompetenz II, welche für die Studierenden des zweiten Semesters vorgesehen war. Das Modul umfasste insgesamt sechs Wochen, aufgerechnet waren dies 12 Stunden. An diesem Seminar haben 45 von 53 eingeschriebenen Studierenden, die in zwei Gruppen aufgeteilt wurden, regelmäßig teilgenommen. Dies ermöglichte einen intensiven und interaktionsstarken Seminarverlauf, in dem genug Zeit gegeben war, auf individuelle Bedürfnisse des Übens einzugehen und über die Erfahrungen im Seminar zu reflektieren.

Das Modul Chorisches Sprechen umfasst vier Phasen, die wie folgt beschrieben werden können: 


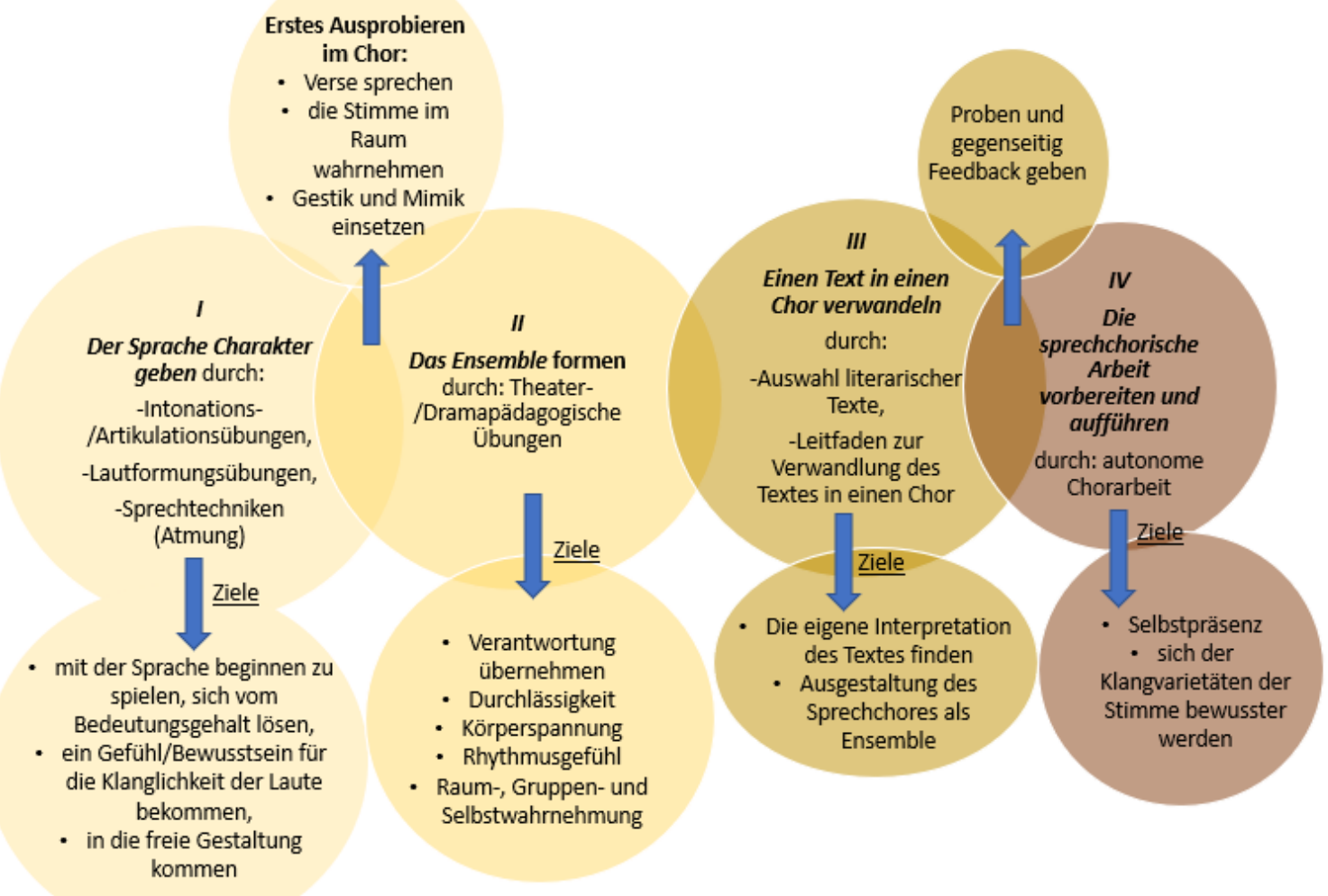

Abbildung 1: Die einzelnen Phasen des Seminarmoduls (Illustration und Idee von L.M.)

Normalerweise werden im ersten und zweiten Semester Präsentationtechniken und Diskussionsstrategien behandelt. Mit diesem neuen Modul jedoch wurden die Studierenden gefordert, sich auf weniger determiniertes und dafür mehr erfahrungsbasiertes Lernen einzulassen. In der Aufbauarbeit des chorischen Sprechens soll bewusst der kognitivsystemische Gehalt von Sprache ausgeschlossen werden, um das halbbewusste Gespür für den Lautklang, den Bildcharakter, den Rhythmus, kurz für das ,Wie' der Sprache und der individuellen Beziehung zu ihr zu verdeutlichen. Es geht im Prinzip um die absichtliche Dekonstruktion des sprachlichen Regelsystems, sodass ein Spielraum mit der Sprache entstehen kann. In diesem neu geschaffenen kreativen Raum liegt das Potenzial, sich der Klanggestalt Zugang zu verschaffen und sie mit der eigenen Stimme zu verbinden. Das ,Wie' der Sprache scheint, seit der pragmatisch-kommunikativen Wende des fremdsprachlichen Unterrichts, verloren gegangen, was auch Bolte (2007) in seinem methodologischen Beitrag stark kritisiert. Er spricht von der „Verredemittlung des Unterrichts“ (S. 2) und sieht die Entwicklung des persönlichen Ausdrucks in der Fremdsprache unterdrückt. 


\subsubsection{Die erste Phase des Seminarmoduls: der Sprache Charakter geben}

Die Kritik am sogenannten kommunikativen Unterricht und am verlorengegangenen Charakter der Sprache bzw. Stimme kann als Ausgangspunkt der Phase 1 genommen werden. Während Bolte (2007) mit alltäglichen Gesprächsfetzen arbeitet, werden in der vorgestellten Seminararbeit, aufgrund des ästhetischen Anspruchs, Verse als Übungsmaterial herangezogen, die mitunter einen hohen Schwierigkeitsgrad der Aussprache, in diesem Fall für Studierende, deren formales Sprachniveau durchchnittlich bei B2 liegt, aufweisen. Punktuell wird auf Sprechtechniken eingegangen, um den Umgang mit komplexen Lautformungen der zungenbrecherähnlichen Verse zu erleichtern. Diesem anwendungsbezogenen Übungsteil geht eine kurze Einführung zur Intonation und Artikulation voraus. Diese sind zwei Grundbestandteile der Ausspracheschulung. An exemplarischen alltagssprachlichen Sätzen werden anschließend gleichzeitig satzmelodische und artikulatorische Fertigkeiten, insbesondere das Binden und Trennen der Laute, sprachpraktisch geübt. Diese Übungen stehen dabei stets im Zeichen des Ausprobierens, wobei auf Regelverläufe, wie die stimmliche Anhebung vor Kommata, aufmerksam gemacht wird.

Damit sich die Sprechenden in der darauffolgenden Stufe vom Gedankeninhalt des Gesprochenen lösen, in das Spiel mit der Sprache kommen und sich mit allen Sinnen auf die Klanglichkeit ihrer eigenen Stimme sowie auf Atmungsverläufe einlassen können, werden Verse ohne semantischen Gehalt, wie in Abb. 2 zu sehen, verwendet.

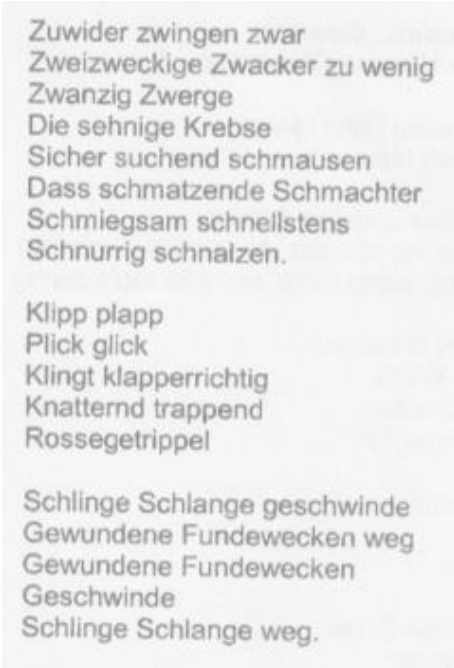

Abbildung 2: Übungen zur Geläufigkeit und Geschicklichkeit aus der Sprachgestaltung (Slezak-Schindler, 2007, S. 140)

Wieder wird im Sinne der Ausspracheschulung auf die Formung der deutsch-spezifischen Laute, wie ,Zw', ,gl', ,pl' und , $\mathrm{kn}^{\prime}$ geachtet und durch einzelnes Sprechen wiederholt. Diese Lautformungsübung dient der plastisch artikulatorischen Verbesserung einerseits, aber andererseits dazu, vor allem ein Gefühl für die charakteristischen Laute zu entwickeln. Die 
Silben gelten dabei als „Träger von Metrum und Melos“ (Berlin Waldorfseminar, o. J., Absatz 4) und rhythmitisieren den Text. Studierenden werden angeregt die Formungen zu übertreiben, also im Lautstrom in den Vordergrund und damit auch ins Bewusstsein zu holen. Genauso sollte auch die Sprechmelodie durch frei gestalterisches Ausprobieren Anklang finden, sodass sich die Sprechenden mehr und mehr vom reproduktiven Sprechen lösen. Die freie Gestaltung verdeutlicht den Lernenden, wie selbstsicher und autonom sie schon mit dem Medium der gesprochenen Sprache umgehen können und inwieweit sie mit Lautformung und Syntaxgliederung vertraut sind. Die inhaltliche Absurdität lädt zudem zum Spiel ein und der normalerweise bestehende Druck nach grammatischer Korrektheit, der oftmals Blockaden verursacht, schwindet. Um ästhetische Erfahrungen zu evozieren, sollte es im Unterricht „darum gehen, über Form im Sinne von grammatischer Kategorie (Adjektiv, Adverb etc.) hinauszudenken“ (Schewe, 2014, S. 174). Nicht zuletzt soll diese Übung dazu beitragen, die eigene Sprechstimme wahrzunehmen und an ihr zu arbeiten.

Ein Wort, eine halbe oder eine ganze Verszeile werden dann auswendig gelernt und in eine Gruppenchorarbeitssequenz gebracht. Ab diesem Arbeitsschritt nehmen sich die Lernenden als Teil eines Stimmen-Ensembles wahr, was in der zweiten Phase vertieft wird. Die Lehrkraft fungiert als Dirigent:in (Bolte, 2007). Hierbei soll auch schon auf die vierte (letzte) Phase Bezug genommen werden, in der diese Rolle entfällt. Zielführend in einem emanzipatorischen Lernprozess ist, dass an letzter Stelle ein Sprechchor mit einer eigenen Stimmenkomposition steht.

Da der Chor sich dort im Seminarraum platziert, wo sich die Lehrkraft in einem regulären Unterricht zeigt - vorne mittig -, ergibt sich für die werdenden Lehrer:innen eine Möglichkeit Selbstpräsenz (Haack, 2018, S. 62) zu üben. Überdies kann mit der eigenen Stimme im Raum, insbesondere mit Lautstärke und stimmlicher Fülle, experimentiert werden. Zu den gesprochenen Versen werden nun auch Gestik und Mimik hinzugenommen, ganz dem individuellen Impuls folgend. Die Konzentration liegt weiterhin auf dem sprachlich-klanglichen und körperlichen Ausdruck und dem Zusammenspiel beider Aspekte. Die Sprechenden übernehmen dabei lediglich einen Satzteil oder eine Wiederholungssequenz, sodass der Vers erst der Reihe nach als Kettenabfolge und, aufbauend darauf, im Wechselspiel inszeniert wird (vgl. dazu die Aufführung eines chorisch inszinierten Textes aus dem Zoran Drvenkars Roman Du von Het Tey, 2017). Außerdem kann der/die Dirigent:in in dieser Aufwärmphase die Sprechenden schon für Rhythmus, (absichtliche) Pausen und allem voran für synchrone Einsätze sensibilisieren (vgl. dazu die Aufführung der TaC Bayreuth, 2017) - all dies zur kreativen Auseinandersetzung mit Sprache und zur Bewusstwerdung der Stimme. Die Überzeichnung und leichte Dramatisierung des Gesprochenen kann zu einer Aushandlung und intentionaler Herausforderung von sprachlichen Verhaltensmustern führen. Letztlich wird in 
der ersten Phase das Sprechwerkzeug Stimme aufgelockert, die Lautformung geschult, ein 'Ensemble-Gespür' entwickelt und die Selbstpräsenz gestärkt.

\subsubsection{Die zweite Phase des Seminarmoduls: das Ensemble}

Ausgangspunkt einer jeden dramapädagogischen Arbeit ist eine Gruppe von Menschen, die unterschiedliche Voraussetzungen (durch Lebenserfahrung/Alter, Geschlecht, sozialkulturelle Herkunft, einsprachige oder mehrsprachige Ausgangssituation und/oder Sprachniveau) mitbringen. Die Zusammenführung eines Chors bedarf eines Trainings, in dem zunächst der Wille zum Spiel ausschlaggebend ist. Das gemeinsame Tun als Chor ist ein multidimensionales Unternehmen, in dem passive mit aktiven, initiative mit zurückhaltenden, selbst-fixierte mit gruppengesteuerten Dispositionen ausbalanciert werden müssen. Die Aushandlung zwischen individuellen Bedürfnissen und denen eines Kollektivs kann mit der Anforderung an die Lehrperson in einem Schulkontext verglichen werden, muss sie doch innerhalb des Lehrkörpers oder als Teil einer Klassengemeinschaft konstruktiv kooperieren. Die Sozialkompetenz als Schlüsselkompetenz für den Lehrerberuf soll in dieser Modulphase stark betont werden.

Der Sprechchor kann nur zu einem guten Ergebnis kommen, wenn jedes einzelne Mitglied volle Verantwortung übernimmt, denn „alle können alles, so gut wie jeder kann“ (Hoffmann, 2006, S. 312 zitiert nach Kirchner, 2016, S. 9). Das gemeinsame Handeln regt außerdem eine Bewusstseinsstufe an, die von Sebastian Nübling als „Bewusst-Außersichsein“ (1998, zitiert nach Kirchner, 2016, S. 9) beschrieben wird. Gemeint sind Weitblick und Durchlässigkeit für die Vorgänge der Umgebung. Auf der eigenen Intuition basierend, gelingt es der Person, selbstgewahr zu sein und gleichzeitig die Ereignisse der direkten Umgebung aufzunehmen und darauf zu reagieren. Letztlich ist dies eine pädagogische Fähigkeit, mit der die Lehrperson als Helfer im Klassenzimmer agiert und dennoch den gesamten Unterrichtsprozess im Blick behält.

Zur Entwicklung des Chores und den o. g. Eigenschaften wurden zwei Übungen aus der Theaterpädagogik (Kirchner, 2016) ausgewählt:

Ü1: Die Übung YOU findet in der Formation eines Kreises satt, in dem durch Klatschen - der Blick ist auf den rechten Partner:in gerichtet - ein deutlich ausgesprochenes ,DU' im Dominoeffekt durch den Kreis gegeben wird. Körperspannung, Konzentration und eine klare Körpersprache sind dabei einzusetzen. Die kollektive Konzentration liegt darin, die Geschwindigkeit zu steigern und wieder zu verringern, bis hin zum Verhallen des Klatschens/Dus. In einer erschwerten Variante kann ein spontaner Richtungswechsel eingebaut werden. Diese Übung wurde gezielt für die Bewusstwerdung der Verantwortung in 
einer Gruppe herangezogen, denn jeder fungiert als ,Perle einer Kette' und ist in dieser Rolle unverzichtbar. Des Weiteren liegt dieser Übung ein Rhythmus zu Grunde, der vom Ensemble aufrechterhalten werden muss. „Jeder einzelne erzeugt und stärkt durch sein Tun diesen Rhythmus, der über die einzelnen hinauswirkt und zwischen den Individuen und der Gruppe eine Verbindung schafft" (Kirchner, 2016, S. 11), sodass in der späteren chorischen Arbeit ein unhörbarer, unterschwelliger Rhythmus zur Organisation und Orientierung als wichtiges Arbeitsprinzip wirkt.

Ü2: Die Plattform oder auch Stop \& Go setzt die Imagination einer Platte voraus, auf der sich alle Teilnehmenden bewegen und die lediglich von einer Nadel gehalten wird. Damit die Platte nicht kippt, ergibt sich die schwierige Aufgabe sich im Raum stets gleichmassig verteilt aufzuhalten, während jeder in Bewegung bleibt. Dies ist eine Übung, den o. g. Weitblick zu schulen und zwischen sich selbst - der eigenen körperlichen Position - und den anderen auszugleichen. Die Wahrnehmung des ganzen Raumes stellt dabei die größte Herausforderung dar. Da die Teilnehmenden die Kontrolle über den ganzen Raum halten sollen, müssen sie körperlich sowie mental alle Ecken des Raumes erfassen.

Beide Phasen sollen an noch ungenügend beleuchtete Aspekte in der Lehrerbildung anknüpfen: Ein Aspekt ist die verbale und körperliche Ausdrucksfähigkeit (Schewe, 1993). Ein weiterer Aspekt ist das persönlichkeitsbildende Arbeitsfeld der Selbst- und Sozialkompetenz, die durch ästhetische Bildungsprozesse angeregt werden können, wie mittlerweile empirisch begründet wurde (vgl. Fleiner, 2016; Haack, 2018; Lutzker, 2007).

\subsubsection{Die dritte Phase des Seminarmoduls: von der Textauswahl zur Chor-Gestaltung}

Sprachbezogener Arbeit liegt zumeist ein Text zu Grunde. Der pädagogischen Sprachgestaltung folgend, „haben ... Märchen und Epen, der Schatz der Dichtung überhaupt, ein hohes sprachliches Niveau. Ihre poetische Struktur, der Sprechrhythmus und die Kraft der Bilder entfalten eine ganzheitliche Gestalt" (Hans, 2018, S. 184). Folglich wurden bewusst literarische Texte herangezogen. Das große Bildungspotenzial solcher Texte soll mit dem neurowissenschaftlichen Forschungsbeitrag Gerald Hüthers betont werden, der in der darin vorzufindenden Bildsprache sozial-ethische Denkprozesse angeregt sieht, die sich auch in neuronalen Verschaltungsmustern im Gehirn abzeichnen und ein ganzes Leben lang wirken (Hans, 2018, S. 183) Fernhin können literarisch-ästhetische Texte dabei unterstützen, den Zugang zum „Affektiven, Emotionalen und Imaginativen“ (Böhme, 2013 zitiert nach Fleiner, 2016, S. 15) zu finden.

Den Studierenden, die sich bereits zu Sprechchören, bestehend aus zwei bis fünf Personen, zusammengefunden hatten, wurde eine Auswahl an prosaischen und poetischen Texten sowie 
zwei Balladen ${ }^{2}$ mit der Anregung unterbreitet, auch selbst literarische Texte aus dieser Gattung vorzulegen. Der zu erarbeitende Text sollte nicht vorgegeben, sondern lediglich in der Gattung und Subgattung eingegrenzt werden, denn die freie Wahl kann motivationsfördernd wirken.

Anhand eines Leitfadens wurden:

1. Textwirkung (Stimmung, Spannungsverlauf) und

2. Verständlichkeit (zentrales Thema, weitere Kernaussagen) textanalytisch herausgearbeitet. Um für die Kraft und Reichhaltigkeit der Sprache und deren Gestaltungmöglichkeiten zu sensibilisieren, gibt es bereits methodologische Hinweise aus der Dramapädagogik (vgl. LiSUM Berlin). In diesem Sinne wurde

3. die mögliche Erzählweise (Tempo, Lautstärke, Pausen, Wiederholungen, Satzüberschneidungen, synchrones Sprechen, paraverbale Effekte wie Lachen, Gähnen, Aufschreien u. a.) innerhalb der Chor-Gruppe in unterschiedlichen Räumen erarbeitet. Als letztes wurde

4. an der Gesamtformation (Dialogisieren, Rollenfestlegen, Formation im Raum/Bühne), auf eine Chor-Performanz hinführend, gefeilt.

Dabei ist der Blick von außen zum Abgleich von Selbst- und Fremdwahrnehmung unverzichtbar: Mitglieder aus anderen Chorgruppen wurden angehalten, einander Feedback zu geben. Dies stellt eine stark kollaborativ-ausgerichtete Arbeitsphase dar, die vor allem die Kritikfähigkeit auf Augenhöhe nahebringt und somit Selbstkompetenz, speziell Selbstwahrnehmung und Selbstreflexion, schult (Haack, 2018, S. 39).

\subsubsection{Die vierte Phase des Seminarmoduls: Vorbereitung und Aufführung der Sprechchöre}

Für die Erläuterung dieser letzten Modulphase soll vorausgeschickt werden, dass am Ende der vorliegenden Sprechchor-Arbeit zwar eine Aufführung steht, dies aber lediglich als Teilziel der Kompetenzbildung gewertet werden kann, denn diese entwickelt sich im künstlerischen Arbeitsprozess (vgl. zur dramatischen Arbeit allgemein Moraitis, 2011). In einer letzten intensiven Probephase, die möglichst autonom in den einzelnen Chor-Gruppen stattfindet und in fließendem Übergang zur vorangegangenen Phase steht, in der zu Austausch bzw. Feedback seitens der anderen Gruppen ermutigt wird, soll eine eigene rhythmisch-klangliche Interpretation des Textes sowie eine Choreografie aus Bewegungsabläufen festgelegt werden. Besonders bedeutungsvoll ist dabei die Bewegung, denn „der Schritt hilft die Sprache zu artikulieren und sie in Silben, in Takt und Rhythmus mit dem ganzen Menschen zu erfassen; er bringt die Sprache in Fluss" (Slezak-Schindler, 2007, S. 23).

\footnotetext{
${ }^{2}$ Die Autor:innen der Texte umfassen Ferdinand Freiligrath, Bertolt Brecht, Johann Wolfgang von Goethe, Reinhard Mey, Hermann Hesse und Hilde Domin.
} 
Die anspruchsvollen literarischen Texte sollten durch Einstudieren verinnerlicht werden, sodass sich die Sprechenden von der Textvorlage Stück für Stück lösen und in einen sprachlichkörperlichen Gestaltungsprozess kommen, insbesondere jedoch sich den Qualitäten ihrer Stimme gewahr werden.

Die Aufführung nun steht als eigene Errungenschaft am Ende dieses Projekts. Die meist stark emotionalisierten Texte, die „tiefste Empfindungen an ein Publikum transportieren können“ (Plath, 2009, S. 28) sind durch die Form der theatralen Mittel geschützt. So können Hemmschwellen und Sprechblockaden überwunden und leise, zurückhaltende Stimmen zu raumfüllenden, klangvollen Stimmen transformiert werden.

Hinsichtlich der Entwicklung von Lehrkompetenz stellt der Bühnenauftritt eine Möglichkeit dar, Selbstpräsenz vor einer Menschengruppe zu üben, was mit Blick auf die pädagogische Professionalisierung von unschätzbarem Wert sein kann. Körpersprache und Stimme sind als Lehrinstrumente zu sehen, die den Raum für das Lernen bereiten.

\section{Resonanz und Ausblick}

Inwieweit konnten Junglehrer:innen persönlichkeitsbezogene Kompetenzen ausbilden und bis zu welchem Grade konnte Lautempfindung angeregt werden? Bolte (2007) versinnbildlicht die Stimme mit einem neuen Kleid, in das Fremdsprachenlernende hineinwachsen und das sie nicht lediglich über das alte Kleid (die Erstsprache) stülpen sollten (S. 2). Inwieweit dieser Identifikationsprozess angestoßen wurde, bleibt weiterhin für Beobachtung und Untersuchung ausstehend. Seitens der Teilnehmenden wurde im Rückblick selbstevaluativ geäußert, dass mehr Kontrolle über die eigene Stimme erlangt, lauteres und deutlicheres Sprechen erzielt, Unsicherheit im Sprechen reduziert und allgemein eine bessere Aussprache festgestellt wurde sowie für die Anwendung performativer Lehr- und Lernansätze im eigenen zukünftigen Unterricht motiviert wurde (persönliche Kommunikation, 25. September 2020). Es ist anzunehmen, dass den Junglehrer:innen Impulse für das dramapädagogische Arbeiten

gegeben und somit Lerntraditionen aufgebrochen werden konnten. Selbstkompetenz als Basiskompetenz (Haack, 2018), die sich eben nicht erst in der eigentlichen Lehrtätigkeit entwickelt, sondern schon im Studium als Teil der Professionalisierung gefördert werden sollte, ist ein Handlungs- und Erfahrungswissensbereich, der m. E. mehr Forschungsinteresse verdient. Fremdsprachenlehrende haben, wie alle anderen Lehrkräfte, einen pädagogischen Auftrag. In diesem gilt es, Zugänge zur Fremdsprache zu schaffen, die diese zur „Freundessprache“ (Kurnatowski, 2009, S. 52) werden lässt und eine identifikative sowie mediative Auseinandersetzung ermöglicht. 
Merkle: Meine neue Stimme

Bibliografie

Anadolu Üniversitesi (o. J.). Speaking Skills I.

http://web.archive.org/web/20210720091859/https://www.anadolu.edu.tr/en/academics/faculti es/course/155623/sozlu-iletisim-becerileri-i-alm/content

Bolte, H. (2007). Soweit die Stimme trägt - Raum für die persönliche Stimme und für Mündliches beim unterrichtlichen Sprach(en)lernen durch Orchestriertes Sprechen. Zeitschrift für Interkulturellen Fremdsprachenunterricht, 12(2), 1-24. https://tujournals.ulb.tudarmstadt.de/index.php/zif/article/view/315/305

Böttger, H. (2005). Englische Ausspracheschulung: Aspekte eines didaktisch-methodischen Designs für die Grundschule. Neusprachliche Mitteilungen aus Wissenschaft und Praxis, 58(1-2), 33-39.

Eichler, W. \& Nold, G. (2007). Sprachbewusstheit. In B. Beck \& E. Klieme. (Hrsg.), Sprachliche Kompetenzen (S. 147-158). Beltz.

Europarat für kulturelle Zusammenarbeit (Hrsg.). (2001). Gemeinsamer europäischer Europäischer Referenzrahmen für Sprachen: lernen, lehren, beurteilen. Langenscheidt. https://www.goethe.de/Z/50/commeuro/20101.htm

Europarat für kulturelle Zusammenarbeit (Hrsg.). (2020). Gemeinsamer europäischer Europäischer Referenzrahmen für Sprachen: lernen, lehren, beurteilen. Begleitband. Langenscheidt.

Fleiner, M. (2016). Performancekünste im Hochschulstudium. Transversale Sprach-, Literatur- und Kulturerfahrungen in der fremdsprachlichen Lehrerbildung. Schibri.

Hans, U. (2018). Sprachgestaltung in der Waldorflehrer-Ausbildung. In H. Kern, T. Zdrazil \& W. M. Götte (Hrsg.), Lehrerbildung für Waldorfschulen (S. 182-194). Beltz-Juventa.

Haack, A. (2018). Dramapädagogik, Selbstkompetenz und Professionalisierung. J.B. Metzler. https://doi.org/10.1007/978-3-658-19951-7

Hekkala, M. (2019). Erfahrungen mit und Entwicklung von Sprachbewusstheit im Umgang mit mehrsprachigem Lehrmaterial - eine Untersuchung bei fortgeschrittenen Deutschlernenden. Universität Helsink.

https://helda.helsinki.fi/bitstream/handle/10138/301919/Hekkala Marjukka Pro gradu 2019.pd f?sequence $=2$ \&isAllowed $=y$

Het Tey. (2017, 23. August). Chorisches Sprechen - Du [Video]. YouTube. https://www.youtube.com/watch?v=ylx5BW5yV2E

Kirchner, C. (2016). „'Wir und: ich und du. Das ist nicht dasselbe." Chorisches Theater als Ensemblekunst (Abschlussarbeit). Theaterpädagogische Akademie Heidelberg.

https://www.theaterwerkstatt-heidelberg.de/wpcontent/uploads/2016/09/tw chorisches theater.pdf

LisUM Berlin-Brandenburg (o. J.). 5. Prüfungkomponente, theaterpädagogische Übungsformen. https://bildungsserver.berlinbrandenburg.de/fileadmin/bbb/unterricht/faecher/sprachen/franzoesisch/sek2/theater 02.pdf 
Lutzker, P. (2007). The Art of foreign language teaching, improvisation and drama in teacher development and language learning. Francke Verlag.

Plath, M. (2009). Biografisches Theater in der Schule: Mit Jugendlichen inszenieren: Darstellendes Spiel in der Sekundarstufe. Beltz Verlag.

Sambanis, M. \& Pickert, K. (2009). Aus-Sprech-Zeit: Spielend üben. Grundschulmagazin Englisch, 7, 912.

Sambanis, M. \& Walter, M. (2019). In Motion: Theaterimpulse zum Sprachenlernen. Von neuesten Befunden der Neurowissenschaft zu konkreten Unterrichtsimpulsen. Cornelsen Scriptor.

Schewe, M. (2014). Für das Ästhetische einen Ort schaffen. DaF als Bauhaus - ein Vorentwurf. In N. Bernstein \& C. Lerchner (Hrsg.), Ästhetisches Lernen im DaF-/Daz-Unterricht. Literatur, Theater, Bildende Kunst, Musik, Film (Materialien Deutsch als Fremdsprache Bd. 93, S. 167-177). Universitätsverlag.

Schewe, M. \& Shaw, P. (Hrsg.). (1993). Towards Drama as a Method in the Foreign Language Classroom. Peter Lang.

Slezak-Schindler, C. (2007). Künstlerisches Sprechen im Schulalter. Grundlegendes für Lehrer und Erzieher, die im Sinne der Erziehungskunst Rudolf Steiners arbeiten (8. Aufl.). Pädagogische Forschungsstelle beim Bund der Freien Waldorfschulen (Edition Waldorf).

Steiner, R. (1983). Von Seelenrätseln. GA 21 Steinerverlag.

Steiner, R. \& Steiner-von Sivers, M. (1981). Sprachgestaltung und Dramatische Kunst. Methodik und Wesen der Sprachgestaltung (4. Aufl.). GA 282 Rudolf Steiner-Nachlaß Verwaltung.

Theater am Campus (TaC). (2017, 24. Januar). Chor der Theaterwissenschaftler:innen [Video]. YouTube. https://www.youtube.com/watch?v=CdysVu9Of-A

Unterweger, E. (2014). Personbezogene überfachliche Kompetenzen von Pädagoginnen und Pädagogen. http://www.oezeps.at/wp-content/uploads/2014/01/Personenbezogene\%C3\%BCberfachliche-Kompetenzen.pdf

Waldorfseminar Berlin (o. J.). Sprachgestaltung. https://waldorfseminar.berlin/waldorflehrerinwerden/studieninhalte/kuenstlerische-prozessarbeit/sprache/\#mainsection 